\title{
A Systematic Design of High-Rate Complex Orthogonal Space-Time Block Codes
}

\author{
Weifeng Su, Member, IEEE, Xiang-Gen Xia, Senior Member, IEEE, and K. J. Ray Liu, Fellow, IEEE
}

\begin{abstract}
In this letter, a systematic design method to generate high-rate space-time block codes from complex orthogonal designs for any number of transmit antennas is proposed. The resulting designs have the best known rates. Two constructions with rates $2 / 3$ and $5 / 8$ are further illustrated for 6 and 7 transmit antennas, respectively.
\end{abstract}

Index Terms-Complex orthogonal designs, diversity, spacetime block codes (STBCs).

\section{INTRODUCTION}

$\mathbf{S}$ PACE-TIME BLOCK CODES (STBCs) from complex orthogonal designs have received extensive interest recently, see for example [1]-[9]. The special structure of orthogonal designs guarantees that these codes achieve full diversity and have a simple maximum-likelihood decoding algorithm.

A complex orthogonal design (COD) in variables $x_{1}, x_{2}, \ldots, x_{k}$ is a $p \times n$ matrix $G$ such that: 1$)$ the entries of $G$ are complex linear combinations of $x_{1}, x_{2}, \ldots, x_{k}$ and their complex conjugates $x_{1}^{*}, x_{2}^{*}, \ldots, x_{k}^{*}$ and 2) the columns of $G$ are orthogonal to each other, i.e.,

$$
G^{\mathcal{H}} G=\left(\left|x_{1}\right|^{2}+\left|x_{2}\right|^{2}+\cdots+\left|x_{k}\right|^{2}\right) I_{n},
$$

where $I_{n}$ is the $n \times n$ identity matrix, and the superscript $\mathcal{H}$ stands for the complex conjugate and transpose of a matrix. The rate of $G$ is defined as $R=k / p$. This design $G$ can be used to generate a STBC for $n$ transmit antennas. The resulting STBC has a rate $k / p$, which means that each codeword with block length $p$ carries $k$ symbols.

The first STBC from COD was proposed in Alamouti [1] for two transmit antennas, which is the following $2 \times 2$ design:

$$
G_{2}=\left[\begin{array}{cc}
x_{1} & x_{2} \\
-x_{2}^{*} & x_{1}^{*}
\end{array}\right] .
$$

Clearly, the rate of $G_{2}$ is 1 . For $n=4$ transmit antennas, there are CODs of rate $R=3 / 4$ [2]-[4], for example,

$$
G_{4}=\left[\begin{array}{cccc}
x_{1} & x_{2} & x_{3} & 0 \\
-x_{2}^{*} & x_{1}^{*} & 0 & x_{3} \\
-x_{3}^{*} & 0 & x_{1}^{*} & -x_{2} \\
0 & -x_{3}^{*} & x_{2}^{*} & x_{1}
\end{array}\right] .
$$

Manuscript received October 1, 2003. The associate editor coordinating the review of this letter and approving it for publication was Prof. H. Jafarkhani. This work was supported in part by the U.S. Army Research Laboratory under Cooperative Agreement DAAD 190120011.

W. Su is with the Department of Electrical and Computer Engineering, University of Maryland, College Park, MD 20742 USA (e-mail: weifeng@ @isr.umd.edu).

X.-G. Xia is with the Department of Electrical and Computer Engineering, University of Delaware, Newark, DE 19716 USA (e-mail: xxia@ee.udel.edu).

K. J. R. Liu is with the Department of Electrical and Computer Engineering, University of Maryland, College Park, MD 20742 USA (e-mail: kjrliu@isr.umd.edu).

Digital Object Identifier 10.1109/LCOMM.2004.827429
The design for $n=3$ transmit antennas is obtained simply by taking the first three columns of $G_{4}$. A class of CODs with rate $R=1 / 2$ was given by Tarokh et al. in [2] by taking advantage of the full-rate real orthogonal designs [2], [10]. Later in [5], two generalized CODs with rates $7 / 11$ and $3 / 5$ were constructed for $n=5$ and $n=6$ transmit antennas, respectively. Recently, a COD with rate $2 / 3$ was presented for $n=5$ transmit antennas [7].

In case of CODs of square size $(p=n)$, it has been shown in [2], [3] that $4 \times 4$ CODs of rate 1 do not exist, and a general result was given in [4] that $R \leq(a+1) /\left(2^{a} b\right)$ if $n=2^{a} b, b$ odd, which is related to the Hurwitz theory [11], [12]. In case of CODs of nonsquare size $(p \geq n)$, it was proved in [8] that rate 1 cannot be achieved for $n \geq 3$ transmit antennas. Later in [6], we showed that for CODs without linear processing, the rate cannot be greater than $3 / 4$ for $n \geq 3$ transmit antennas. Further in [9], it was showed that this result holds for any COD.

In this letter, we first present two hand-crafted high-rate CODs for $n=6$ and $n=7$ transmit antennas explicitly. Then we propose a general algorithm to generate high-rate CODs for any number of transmit antennas.

\section{CODS FOR ANY NUMBER OF TRANSMIT ANTENNAS}

For $n=6$ transmit antennas, we construct a $30 \times 6 \mathrm{COD}$ with rate $R=2 / 3$ as shown in matrix $G_{6}$, at the left-hand column of the next page. This design contains $k=20$ symbols and has a block length or delay $p=30$. For $n=7$ transmit antennas, a $56 \times 7 \mathrm{COD} G_{7}$ is specified as shown in the matrix, shown at the right-hand column of the next page. This design contains $k=35$ symbols and has a block length $p=56$. Clearly, the rate of $G_{7}$ is $R=k / p=5 / 8$.

The basic methodology of constructing the orthogonal designs $G_{6}$ and $G_{7}$ is stated as follows. For convenience of explanation, let us say a row in an orthogonal design is conjugate (nonconjugate) if all symbols except zeros in this row have (do not have) complex conjugate. For example, the first row of the orthogonal design $G_{2}$ in (2) is nonconjugate, and the second row is conjugate. We started from $G_{1}=x_{1} I_{1}$, and generated $G_{m}$ from $G_{m-1}$ iteratively for any $2 \leq m \leq n$. In each iteration from $G_{m-1}$ to $G_{m}$, we added a new column with some new symbols. The number of the new symbols and the position of each new symbol in the new column depend on the numbers of conjugate rows and nonconjugate rows in $G_{m-1}$. If the number of nonconjugate rows is not less than that of conjugate rows, we add some new symbols into the nonconjugate rows and set some zeros in the conjugate rows. If the number of nonconjugate rows is less than that of conjugate rows, we put some new symbols with complex conjugate into the conjugate rows 


$$
G_{6}=\left[\begin{array}{cccccc}
x_{1} & x_{2} & x_{3} & 0 & x_{7} & 0 \\
-x_{2}^{*} & x_{1}^{*} & 0 & x_{4}^{*} & 0 & x_{11}^{*} \\
-x_{3}^{*} & 0 & x_{1}^{*} & x_{5}^{*} & 0 & x_{12}^{*} \\
0 & -x_{3}^{*} & x_{2}^{*} & x_{6}^{*} & 0 & x_{13}^{*} \\
0 & -x_{4} & -x_{5} & x_{1} & x_{8} & 0 \\
x_{4} & 0 & -x_{6} & x_{2} & x_{9} & 0 \\
x_{5} & x_{6} & 0 & x_{3} & x_{10} & 0 \\
-x_{6}^{*} & x_{5}^{*} & -x_{4}^{*} & 0 & 0 & x_{14}^{*} \\
-x_{7}^{*} & 0 & 0 & -x_{8}^{*} & x_{1}^{*} & x_{15}^{*} \\
0 & -x_{7}^{*} & 0 & -x_{9}^{*} & x_{2}^{*} & x_{16}^{*} \\
0 & 0 & -x_{7}^{*} & -x_{10}^{*} & x_{3}^{*} & x_{17}^{*} \\
-x_{9}^{*} & x_{8}^{*} & 0 & 0 & x_{4}^{*} & x_{18}^{*} \\
-x_{10}^{*} & 0 & x_{8}^{*} & 0 & x_{5}^{*} & x_{19}^{*} \\
0 & -x_{10}^{*} & x_{9}^{*} & 0 & x_{6}^{*} & x_{20}^{*} \\
x_{8} & x_{9} & x_{10} & -x_{7} & 0 & 0 \\
0 & -x_{11} & -x_{12} & 0 & -x_{15} & x_{1} \\
x_{11} & 0 & -x_{13} & 0 & -x_{16} & x_{2} \\
x_{12} & x_{13} & 0 & 0 & -x_{17} & x_{3} \\
0 & 0 & x_{14} & -x_{11} & -x_{18} & x_{4} \\
0 & -x_{14} & 0 & -x_{12} & -x_{19} & x_{5} \\
x_{14} & 0 & 0 & -x_{13} & -x_{20} & x_{6} \\
x_{15} & x_{16} & x_{17} & 0 & 0 & x_{7} \\
0 & -x_{18} & -x_{19} & x_{15} & 0 & x_{8} \\
x_{18} & 0 & -x_{20} & x_{16} & 0 & x_{9} \\
x_{19} & x_{20} & 0 & x_{17} & 0 & x_{10} \\
-x_{13}^{*} & x_{12}^{*} & -x_{11}^{*} & -x_{14}^{*} & 0 & 0 \\
-x_{16}^{*} & x_{15}^{*} & 0 & x_{18}^{*} & -x_{11}^{*} & 0 \\
-x_{17}^{*} & 0 & x_{15}^{*} & x_{19}^{*} & -x_{12}^{*} & 0 \\
0 & -x_{17}^{*} & x_{16}^{*} & x_{20}^{*} & -x_{13}^{*} & 0 \\
x_{20}^{*} & -x_{19}^{*} & x_{18}^{*} & 0 & x_{14}^{*} & 0
\end{array}\right]
$$

and set some zeros in the nonconjugate rows accordingly. Due to the new symbols in the $m$ th column, we have to arrange some additional rows to guarantee the orthogonality.

With the basic ideas, we have developed a general algorithm as follows. Denote $k_{m-1}$ and $p_{m-1}$, respectively, the numbers of symbols and rows in $G_{m-1}$ for any $m \geq 2$.

- Initialization: Start from $G_{1}=x_{1} I_{1}$.

- Generate $G_{m}$ from $G_{m-1}$ : for $m=2,3, \ldots, n$

1) Calculate $\nu_{0}$ and $\nu_{1}$, the numbers of nonconjugate rows and conjugate rows in $G_{m-1}$, respectively.

2) Add some new symbols into the $m$ th column from the first row to the $p_{m-1}$ th row. If $\nu_{0} \geq \nu_{1}$, then add new symbols $x_{k_{m-1}+1}, \ldots, x_{k_{m-1}+\nu_{0}}$ into the nonconjugate rows, and set zeros into the conjugate rows; If $\nu_{0}<\nu_{1}$, then add new symbols with complex conjugate $x_{k_{m-1}+1}^{*}, \ldots, x_{k_{m-1}+\nu_{1}}^{*}$ into the conjugate rows, and set zeros into the nonconjugate rows.

3) Since the symbols $x_{1}, x_{2}, \ldots, x_{k_{m-1}}$ must appear in each column, we further specify the entries in the $m$ th column after the $p_{m-1}$ th row as follows. If $\nu_{0} \geq \nu_{1}\left(\right.$ or $\left.\nu_{0}<\nu_{1}\right)$, then put $x_{1}^{*}, x_{2}^{*}, \ldots, x_{k_{m-1}}^{*}$ (or $x_{1}, x_{2}, \ldots, x_{k_{m-1}}$ ) into the $m$ th column from the $\left(p_{m-1}+1\right)$ th row to the $\left(p_{m-1}+k_{m-1}\right)$ th row.

4) According to the orthogonality of the $m$ th column to each of the first $m-1$ columns, we fill the entries

\begin{tabular}{|c|c|c|c|c|c|c|}
\hline$x_{1}$ & $x_{2}$ & $x_{3}$ & 0 & $x_{7}$ & 0 & $x_{21}$ \\
\hline$-x_{2}^{*}$ & $x_{1}^{*}$ & 0 & $x_{4}^{*}$ & 0 & $x_{11}^{*}$ & 0 \\
\hline$-x_{3}^{*}$ & 0 & $x_{1}^{*}$ & $x_{5}^{*}$ & 0 & $x_{12}^{*}$ & 0 \\
\hline 0 & $-x_{3}^{*}$ & $x_{2}^{*}$ & $x_{6}^{*}$ & 0 & $x_{13}^{*}$ & 0 \\
\hline 0 & $-x_{4}$ & $-x_{5}$ & $x_{1}$ & $x_{8}$ & 0 & $x_{22}$ \\
\hline$x_{4}$ & 0 & $-x_{6}$ & $x_{2}$ & $x_{9}$ & 0 & $x_{23}$ \\
\hline$x_{5}$ & $x_{6}$ & 0 & $x_{3}$ & $x_{10}$ & 0 & $x_{24}$ \\
\hline$-x_{6}^{*}$ & $x_{5}^{*}$ & $-x_{4}^{*}$ & 0 & 0 & $x_{14}^{*}$ & 0 \\
\hline$-x_{7}^{*}$ & 0 & 0 & $-x_{8}^{*}$ & $x_{1}^{*}$ & $x_{15}^{*}$ & 0 \\
\hline 0 & $-x_{7}^{*}$ & 0 & $-x_{9}^{*}$ & $x_{2}^{*}$ & $x_{16}^{*}$ & 0 \\
\hline 0 & 0 & $-x_{7}^{*}$ & $-x_{10}^{*}$ & $x_{3}^{*}$ & $x_{17}^{*}$ & 0 \\
\hline$-x_{9}^{*}$ & $x_{8}^{*}$ & 0 & 0 & $x_{4}^{*}$ & $x_{18}^{*}$ & 0 \\
\hline$-x_{10}^{*}$ & 0 & $x_{8}^{*}$ & 0 & $x_{5}^{*}$ & $x_{19}^{*}$ & 0 \\
\hline 0 & $-x_{10}^{*}$ & $x_{9}^{*}$ & 0 & $x_{6}^{*}$ & $x_{20}^{*}$ & 0 \\
\hline$x_{8}$ & $x_{9}$ & $x_{10}$ & $-x_{7}$ & 0 & 0 & $x_{25}$ \\
\hline 0 & $-x_{11}$ & $-x_{12}$ & 0 & $-x_{15}$ & $x_{1}$ & $x_{26}$ \\
\hline$x_{11}$ & 0 & $-x_{13}$ & 0 & $-x_{16}$ & $x_{2}$ & $x_{27}$ \\
\hline$x_{12}$ & $x_{13}$ & 0 & 0 & $-x_{17}$ & $x_{3}$ & $x_{28}$ \\
\hline 0 & 0 & $x_{14}$ & $-x_{11}$ & $-x_{18}$ & $x_{4}$ & $x_{29}$ \\
\hline 0 & $-x_{14}$ & 0 & $-x_{12}$ & $-x_{19}$ & $x_{5}$ & $x_{30}$ \\
\hline$x_{14}$ & 0 & 0 & $-x_{13}$ & $-x_{20}$ & $x_{6}$ & $x_{31}$ \\
\hline$x_{15}$ & $x_{16}$ & $x_{17}$ & 0 & 0 & $x_{7}$ & $x_{32}$ \\
\hline 0 & $-x_{18}$ & $-x_{19}$ & $x_{15}$ & 0 & $x_{8}$ & $x_{33}$ \\
\hline$x_{18}$ & 0 & $-x_{20}$ & $x_{16}$ & 0 & $x_{9}$ & $x_{34}$ \\
\hline$x_{19}$ & $x_{20}$ & 0 & $x_{17}$ & 0 & $x_{10}$ & $x_{35}$ \\
\hline$-x_{13}^{*}$ & $x_{12}^{*}$ & $-x_{11}^{*}$ & $-x_{14}^{*}$ & 0 & 0 & 0 \\
\hline$-x_{16}^{*}$ & $x_{15}^{*}$ & 0 & $x_{18}^{*}$ & $-x_{11}^{*}$ & 0 & 0 \\
\hline$-x_{17}^{*}$ & 0 & $x_{15}^{*}$ & $x_{19}^{*}$ & $-x_{12}^{*}$ & 0 & 0 \\
\hline 0 & $-x_{17}^{*}$ & $x_{16}^{*}$ & $x_{20}^{*}$ & $-x_{13}^{*}$ & 0 & 0 \\
\hline$x_{20}^{*}$ & $-x_{19}^{*}$ & $x_{18}^{*}$ & 0 & $x_{14}^{*}$ & 0 & 0 \\
\hline$-x_{21}^{*}$ & 0 & 0 & $-x_{22}^{*}$ & 0 & $-x_{26}^{*}$ & $x_{1}^{*}$ \\
\hline 0 & $-x_{21}^{*}$ & 0 & $-x_{23}^{*}$ & 0 & $-x_{27}^{*}$ & $x_{2}^{*}$ \\
\hline 0 & 0 & $-x_{21}^{*}$ & $-x_{24}^{*}$ & 0 & $-x_{28}^{*}$ & $x_{3}^{*}$ \\
\hline$-x_{23}^{*}$ & $x_{22}^{*}$ & 0 & 0 & 0 & $-x_{29}^{*}$ & $x_{4}^{*}$ \\
\hline$-x_{24}^{*}$ & 0 & $x_{22}^{*}$ & 0 & 0 & $-x_{30}^{*}$ & $x_{5}^{*}$ \\
\hline 0 & $-x_{24}^{*}$ & $x_{23}^{*}$ & 0 & 0 & $-x_{31}^{*}$ & $x_{6}^{*}$ \\
\hline 0 & 0 & 0 & $x_{25}^{*}$ & $-x_{21}^{*}$ & $-x_{32}^{*}$ & $x_{7}^{*}$ \\
\hline$-x_{25}^{*}$ & 0 & 0 & 0 & $-x_{22}^{*}$ & $-x_{33}^{*}$ & $x_{8}^{*}$ \\
\hline 0 & $-x_{25}^{*}$ & 0 & 0 & $-x_{23}^{*}$ & $-x_{34}^{*}$ & $x_{9}^{*}$ \\
\hline 0 & 0 & $-x_{25}^{*}$ & 0 & $-x_{24}^{*}$ & $-x_{35}^{*}$ & $x_{10}^{*}$ \\
\hline$-x_{27}^{*}$ & $x_{26}^{*}$ & 0 & $x_{29}^{*}$ & 0 & 0 & $x_{11}^{*}$ \\
\hline$-x_{28}^{*}$ & 0 & $x_{26}^{*}$ & $x_{30}^{*}$ & 0 & 0 & $x_{12}^{*}$ \\
\hline 0 & $-x_{28}^{*}$ & $x_{27}^{*}$ & $x_{31}^{*}$ & 0 & 0 & $x_{13}^{*}$ \\
\hline$-x_{31}^{*}$ & $x_{30}^{*}$ & $-x_{29}^{*}$ & 0 & 0 & 0 & $x_{14}^{*}$ \\
\hline$-x_{32}^{*}$ & 0 & 0 & $-x_{33}^{*}$ & $x_{26}^{*}$ & 0 & $x_{15}^{*}$ \\
\hline 0 & $-x_{32}^{*}$ & 0 & $-x_{34}^{*}$ & $x_{27}^{*}$ & 0 & $x_{16}^{*}$ \\
\hline 0 & 0 & $-x_{32}^{*}$ & $-x_{35}^{*}$ & $x_{28}^{*}$ & 0 & $x_{17}^{*}$ \\
\hline$-x_{34}^{*}$ & $x_{33}^{*}$ & 0 & 0 & $x_{29}^{*}$ & 0 & $x_{18}^{*}$ \\
\hline$-x_{35}^{*}$ & 0 & $x_{33}^{*}$ & 0 & $x_{30}^{*}$ & 0 & $x_{19}^{*}$ \\
\hline 0 & $-x_{35}^{*}$ & $x_{34}^{*}$ & 0 & $x_{31}^{*}$ & 0 & $x_{20}^{*}$ \\
\hline$x_{22}$ & $x_{23}$ & $x_{24}$ & $-x_{21}$ & $-x_{25}$ & 0 & 0 \\
\hline$x_{26}$ & $x_{27}$ & $x_{28}$ & 0 & $x_{32}$ & $-x_{21}$ & 0 \\
\hline 0 & $-x_{29}$ & $-x_{30}$ & $x_{26}$ & $x_{33}$ & $-x_{22}$ & 0 \\
\hline$x_{29}$ & 0 & $-x_{31}$ & $x_{27}$ & $x_{34}$ & $-x_{23}$ & 0 \\
\hline$x_{30}$ & $x_{31}$ & 0 & $x_{28}$ & $x_{35}$ & $-x_{24}$ & 0 \\
\hline & $-x_{3}$ & $-x_{35}$ & $x_{32}$ & 0 & $r_{0 r}$ & 0 \\
\hline
\end{tabular}

of the first $m-1$ columns from the $\left(p_{m-1}+1\right)$ th row to the $\left(p_{m-1}+k_{m-1}\right)$ th row as follows. 


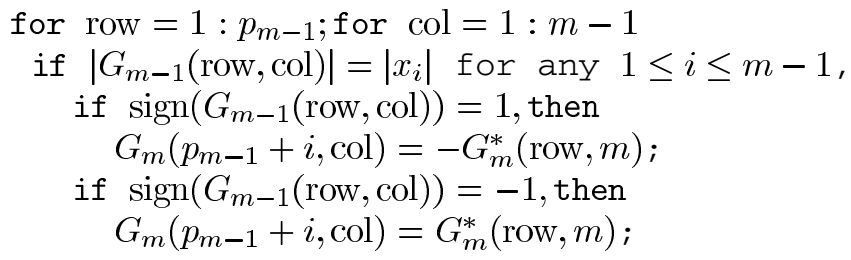
end ;

Set zeros to those unspecified entries;

5) After Step 4, the $m$ th column is ready. However, we need to arrange some additional rows to ensure the orthogonality of the first $m-1$ columns. In the additional rows, simply put zeros at the $m$ th column.

for $\begin{aligned} & \text { Set RowCount } \\ & \text { row }=p_{m-1}+1\end{aligned} p_{m-1}+p_{m-1}+k_{m-1}$.

if $G_{m}($ row, col1 $) \neq 0$ and $G_{m}($ row, col2 $) \neq 0$ for

$1 \leq \operatorname{col} 1<\operatorname{col} 2 \leq m-1$, then

Set NeedNewRow $=$ TRUE;

$X_{11}=G_{m}($ row, col1 $) ; X_{12}=G_{m}($ row, col2 $)$;

for NewRow $=p_{m-1}+k_{m-1}+1$ : RowCount

$X_{21}=G_{m}($ NewRow, col1) ;

$X_{22}=G_{m}$ (NewRow, col2)；

if $X_{21}=0$ and $\left|X_{22}\right|=\left|X_{11}\right|$, then

if $X_{11}=X_{22}^{*}$, then

$G_{m}($ NewRow, col1 $)=-X_{12}^{*} ;$

if $X_{11}=-X_{22}^{*}$, then

$G_{m}($ NewRow, col1 $)=X_{12}^{*}$;

NeedNewRow $=$ FALSE ;

if $\left|X_{21}\right|=\left|X_{12}\right|$ and $X_{22}=0$, then

if $X_{21}=X_{12}^{*}$, then

$G_{m}($ NewRow, $\operatorname{col} 2)=-X_{11}^{*} ;$

if $X_{21}=-X_{12}^{*}$, then

$G_{m}($ NewRow, col1 $)=X_{11}^{*}$;

NeedNewRow $=$ FALSE ;

if $\left|X_{21}\right|=\left|X_{12}\right|$ and $\left|X_{22}\right|=\left|X_{11}\right|$, then

NeedNewRow $=$ FALSE ;

end;

if NeedNewRow = TRUE, then

RowCount $=$ RowCount +1 ;

$G_{m}($ RowCount, col1 $)=-G_{m}^{*}($ row, $\operatorname{col} 2)$;

$G_{m}($ RowCount, col2 $)=G_{m}^{*}($ row, col1 $)$;

end ;

- Let $k_{m}=k_{m-1}+\max \left(\nu_{0}, \nu_{1}\right), p_{m}=$ RowCount, and repeat the above iteration if $m<n$.

By Steps 4 and 5, the orthogonality of the resulting design is guaranteed. This algorithm can be used to generate CODs for any number of transmit antennas. In Table I, we list the number of symbols, block length and rate of CODs for $2 \leq n \leq 18$ transmit antennas. We observe from the table that for $n \leq 18$, the rate of the obtained design satisfies a rule of $\left(n_{0}+1\right) /\left(2 n_{0}\right)$ if $n=2 n_{0}$ or $n=2 n_{0}-1$, which is conjectured optimal in [9] for any $n$. We believe that the proposed algorithm will continue to generate CODs with the optimal rate for $n>18$.

\section{CONCLUSION AND DISCUSSION}

We presented in this letter a systematic design method to generate high-rate complex orthogonal STBCs for any number of
TABLE I

High-RATE STBCs From COMPLEX ORTHOGONAL DESIGNS FOR $2 \leq n \leq 18$ TRANSMIT ANTENNAS

\begin{tabular}{l|c|c|c}
\hline & $\begin{array}{c}\text { Symbols } \\
k\end{array}$ & $\begin{array}{c}\text { Block Length } \\
p\end{array}$ & $\begin{array}{c}\text { Rate } \\
k / p\end{array}$ \\
\hline$n=2$ & 2 & 2 & 1 \\
$n=3$ & 3 & 4 & $3 / 4$ \\
$n=4$ & 6 & 8 & $3 / 4$ \\
$n=5$ & 10 & 15 & $2 / 3$ \\
$n=6$ & 20 & 30 & $2 / 3$ \\
$n=7$ & 35 & 56 & $5 / 8$ \\
$n=8$ & 70 & 112 & $5 / 8$ \\
$n=9$ & 126 & 210 & $3 / 5$ \\
$n=10$ & 252 & 420 & $3 / 5$ \\
$n=11$ & 462 & 792 & $7 / 12$ \\
$n=12$ & 924 & 1584 & $7 / 12$ \\
$n=13$ & 1716 & 3003 & $4 / 7$ \\
$n=14$ & 3432 & 6006 & $4 / 7$ \\
$n=15$ & 6435 & 11440 & $9 / 16$ \\
$n=16$ & 12870 & 22880 & $9 / 16$ \\
$n=17$ & 24310 & 43758 & $5 / 9$ \\
$n=18$ & 48620 & 87516 & $5 / 9$ \\
\hline
\end{tabular}

transmit antennas. Also, the designs for $n \leq 18$ transmit antennas were obtained and two designs with rates $2 / 3$ and $5 / 8$ were further illustrated for 6 and 7 transmit antennas, respectively. All of the resulting CODs have the best known rates that are conjectured optimal. Moreover, these designs indicate that CODs with nonsquare size do provide larger rates than those with square size. For example, for $n=8$ transmit antennas, the maximum rate of CODs with square size is $1 / 2$ [4] while the COD obtained here has a rate of $5 / 8$. This phenomenon was observed in [2] in case of real orthogonal designs.

Note that for $n>10$ transmit antennas, the block length of the obtained CODs is large. For example, for $n=16$ transmit antennas, the block length is $p=22880$. Thus, in this case, the resulting COD is more of theoretical interest.

\section{REFERENCES}

[1] S. Alamouti, "A simple transmit diversity technique for wireless communications," in IEEE J. Select. Areas Commun., vol. 16, Oct. 1998, pp. $1451-1458$.

[2] V. Tarokh, H. Jafarkhani, and A. R. Calderbank, "Space-time block codes from orthogonal designs," IEEE Trans. Inform. Theory, vol. 45, pp. 1456-1467, Oct. 1999.

[3] G. Ganesan and P. Stoica, "Space-time block codes: A maximum SNR approach," IEEE Trans. Inform. Theory, pp. 1650-1656, Apr. 2001.

[4] O. Tirkkonen and A. Hottinen, "Square-matrix embeddable space-time block codes for complex signal constellations," IEEE Trans. Inform. Theory, vol. 48, pp. 1122-1126, Feb. 2002.

[5] W. Su and X.-G. Xia, "Two generalized complex orthogonal space-time block codes of rates $7 / 11$ and $3 / 5$ for 5 and 6 transmit antennas," IEEE Trans. Inform. Theory, vol. 49, pp. 313-316, Jan. 2003.

[6] - "On space-time block codes from complex orthogonal designs," Wireless Personal Communications, vol. 25, no. 1, pp. 1-26, Apr. 2003.

[7] X.-B. Liang, "A high-rate orthogonal space-time block code," IEEE Commun. Lett., vol. 7, pp. 222-223, May 2003.

[8] X.-B. Liang and X.-G. Xia, "On the nonexistence of rate-one generalized complex orthogonal designs," IEEE Inform. Theory, to be published.

[9] H. Wang and X.-G. Xia, "Upper bounds of rates of space-time block codes from complex orthogonal designs," IEEE Inform. Theory, vol. 49, pp. 2788-2796, Oct. 2003.

[10] A. V. Geramita and J. Seberry, Orthogonal Designs, Quadratic Forms, and Hadamard Matrices, Lecture Notes in Pure and Applied Mathematics. New York: Marcel Dekker, 1979, vol. 43.

[11] A. V. Geramita and J. M. Geramita, "Complex orthogonal designs," J. Combinatorial Theory, ser. Series A (25), pp. 211-225, 1978.

[12] D. B. Shapiro, Compositions of Quadratic Forms. New York: De Gruyter, 2000. 